\title{
Electronic origin of void formation in fec metals
}

\author{
Xu Zhang and Gang Lu* \\ Department of Physics and Astronomy, California State University Northridge, California 91330-8268, USA
}

(Received 19 November 2007; revised manuscript received 8 April 2008; published 2 May 2008)

\begin{abstract}
Voids can be formed by supersaturation of vacancies created by energetic particle irradiation, quenching, or mechanical deformation. By using multiscale simulations, we studied the thermodynamic processes of void formation in Al. We discovered an important connection between vacancy-induced bonding cage and the tendency of void formation in fcc metals. A model is proposed to explain the observed connection, linking the electronic structure to the free energy of a void nucleus. We found that the formation of the bonding cages is closely related to the directionality of the chemical bonding in fcc metals.
\end{abstract}

DOI: 10.1103/PhysRevB.77.174102

PACS number(s): 61.72.Qq, 65.40.G-, 71.55.-i

\section{INTRODUCTION}

Voids can be formed in a supersaturated vacancy atmosphere created by irradiation, electromigration, or mechanical deformation. Once formed, voids can significantly degrade the performance of materials: voids can lead to macroscopic swelling in fusion reactor materials; $;^{1,2}$ electromigration-induced voids can result in open circuits, hillocks, and other failures in microelectronic components; 3,4 void formation is also the crucial precursor for dynamical fractures of ductile materials. ${ }^{5}$ Despite the importance of void formation in many aspects of materials science and engineering, its microscopic mechanism remains poorly understood. The well-established classical nucleation theory has been widely used in studying void formation. ${ }^{6-8}$ Such a theory can give a precise thermodynamic description of void formation, evolving from an embryo (one or two vacancies) to a nucleus with a critical size (seven or eight vacancies) and to a void. Although impurities and other inhomogeneities in a crystal lattice such as grain boundaries, dislocations, and phase boundaries play important roles in void formation (termed as heterogeneous nucleation), in this paper, we focus on the homogeneous nucleation ${ }^{9,10}$ in pure and perfect crystals. In the present paper, a void refers to a large three dimensional vacancy cluster, which could contain thousands of lattice vacancies.

In order to employ the classical homogeneous nucleation theory, care must be taken to obtain reliable free energies for vacancy clusters. ${ }^{11}$ Unfortunately, conventional computational approaches cannot provide sufficiently accurate energetics for the relevant vacancy clusters. For example, although first-principles calculations are rather accurate in terms of energetics, they can only treat very small vacancy clusters due to their significant computational expense. On the other hand, while empirical atomistic simulations are much cheaper in terms of computational expense, they are, in general, not accurate enough to predict the important energetics for large vacancy clusters. ${ }^{12-15}$ To overcome the aforementioned difficulties, we employ a multiscale approach ${ }^{16,17}$ that combines the first-principles quantum mechanical calculations with classical atomistic simulations to compute the energetics of the vacancy clusters.

In this paper, we report on the electronic origin of void formation in fcc metals. We find a one-to-one correspon- dence between a negative binding energy for a divacancy and the formation of a bonding cage around a monovacancy in all fcc metals. We discover that the bonding cage plays a crucial role in void formation and the electronic origin of void formation is the same for all fcc metals. A connection between the propensity of void formation and the electronic structure of the materials is established, which could be exploited to tailor the properties of the materials for novel applications.

\section{COMPUTATIONAL METHODS}

According to the steady-state nucleation theory, ${ }^{18,19}$ the concentration of vacancy clusters with various sizes can be determined by the Gibbs free energy (consisting of formation enthalpy, formation entropy, and configurational entropy) under equilibrium between cluster sizes with the constraint of a constant supersaturation..$^{20}$ At a constant pressure $P$, the vacancy formation enthalpy can be approximated well by the formation energy owing to the large supercell used in the calculations. ${ }^{21,22}$ The formation energy of an $n$-vacancy cluster can be calculated as ${ }^{21-25}$

$$
E_{n v}^{F}=E_{n v}^{\mathrm{tot}}(N-n)-\frac{N-n}{N} E^{\mathrm{tot}}(N),
$$

where $E_{n v}^{\mathrm{tot}}(N-n)$ is the total energy of the supercell containing $N-n$ atoms with an $n$-vacancy cluster, and $E^{\text {tot }}(N)$ is the total energy of the supercell containing $N$ atoms in the perfect bulk configuration. The vacancy formation entropy $S_{n v}^{F}$ can be analogously defined. It was found that the formation enthalpy increases very slowly with temperature $T$ when $T$ is less than $700 \mathrm{~K} .{ }^{24}$ For the formation entropy, in addition to the vibrational entropy that is considered in the present work, there is also a minor $T$-dependent electronic contribution to the entropy, which is negligible. ${ }^{26}$ Therefore, the temperature dependence of $E_{n \mathrm{v}}^{F}$ and $S_{n \mathrm{v}}^{F}$ is neglected in the present paper. We determine the Gibbs binding free energy at temperature $T$ as

$$
G_{n v}^{B}=E_{n v}^{B}-T S_{n v}^{B},
$$

with the binding energy and binding entropy of the $n$-vacancy cluster given by 


$$
E_{n v}^{B}=n E_{1 v}^{F}-E_{n v}^{F}
$$

and

$$
S_{n v}^{B}=n S_{1 v}^{F}-S_{n v}^{F},
$$

respectively. The configurational entropy has two contributions: One is the entropy of mixing taking into account the possibilities of $n_{\mathrm{vac}}$ vacancies occupying $N_{\text {latt }}$ lattice sites, which results in the well-known expression of vacancy concentration [for example, Eq. (8)]. The other one comes from counting the possible configurations of a given vacancy cluster. However, in this paper, we ignore the second contribution by considering only the vacancy cluster for a given $n$, which minimizes the number of broken bonds. This approximation is motivated by the fact that the vacancy formation energy decreases with decreasing number of broken bonds. The positive (negative) value of $G_{n v}^{B}$ indicates that the $n$-vacancy cluster is thermodynamically stable (unstable) against a simultaneous dissociation into $n$ monovacancies. In the following, we focus on aluminum as an example to illustrate the general properties of fcc metals.

We employ multiscale simulations ${ }^{16,17}$ to calculate the vacancy binding energy (the dominant term in the Gibbs binding free energy). The multiscale approach concurrently combines quantum mechanical and empirical atomistic calculations, and it can treat up to a few tens of vacancies with the desired quantum accuracy. In this approach, the entire system of interest is partitioned into an orbital-free density function theory (OFDFT) region and an embedded-atom method (EAM) region, where the OFDFT (Refs. 27-29) and the empirical EAM (Ref. 30) calculations are carried out, respectively. The OFDFT region (region I) is embedded in a much larger EAM region (region II) so that the latter provides an appropriate boundary condition for the former. The interaction energy between regions I and II is quantum mechanically calculated with the OFDFT energy formulation. The total energy of the system is thus expressed as ${ }^{16,17}$

$$
\begin{aligned}
E_{\text {tot }}\left[\mathbf{R}^{\text {tot }}\right]= & \min _{\rho^{\mathrm{I}}}\left\{E_{\mathrm{OFDFT}}\left[\rho^{\mathrm{I}} ; \mathbf{R}^{\mathrm{I}}\right]+E_{\mathrm{OFDFT}}^{\mathrm{int}}\left[\rho^{\mathrm{I}}, \rho^{\mathrm{II}} ; \mathbf{R}^{\mathrm{I}}, \mathbf{R}^{\mathrm{II}}\right]\right\} \\
& +E_{\mathrm{EAM}}\left[\mathbf{R}^{\mathrm{II}}\right]-\sum_{i \in \mathrm{II}} \Delta \mathbf{F}_{i}^{\mathrm{corr}} \cdot \Delta \mathbf{R}_{i},
\end{aligned}
$$

where $\mathbf{R}^{\text {tot }} \equiv \mathbf{R}^{\mathrm{I}} \cup \mathbf{R}^{\mathrm{II}}$ and $\rho^{\mathrm{I}}\left(\mathbf{R}^{\mathrm{I}}\right)$ and $\rho^{\mathrm{II}}\left(\mathbf{R}^{\mathrm{II}}\right)$ represent the charge densities (atomic positions) associated with regions I and II, respectively. The first three terms in Eq. (5) represent the energy in region I, the interaction energy between regions I and II, and the energy in region II, respectively. The last term is the work done by the correctional force $\Delta \mathbf{F}_{i}^{\text {corr }}$, which is introduced to eliminate the force mismatch at the OFDFT/ EAM interface. ${ }^{17,31} \Delta \mathbf{R}_{i}$ is the displacement of atoms in region II during the relaxation. For the OFDFT, we use the Wang-Govind-Carter kinetic energy functional with a density-dependent

kernel and parameters $\left\{\alpha, \beta, \gamma, \rho^{*}\right\}=\{5 / 6+\sqrt{5} / 6,5 / 6$ $\left.-\sqrt{5} / 6,2.7,0.183 \AA^{-3}\right\} .{ }^{29}$ The Al ions are represented with the Goodwin-Needs-Heine local pseudopotential. ${ }^{32}$ The Perdew-Zunger local density approximation ${ }^{33}$ (LDA) is used to evaluate the exchange-correlation functional. More details of the multiscale method can be found in Refs. 16 and 17.
To calculate the vacancy binding energy in $\mathrm{Al}$, we choose a large $14 a_{0} \times 14 a_{0} \times 14 a_{0}$ supercell, with $a_{0}$ being the lattice constant of bulk Al. The supercell contains $N=10976$ atoms and is partitioned into an OFDFT region (256 atoms) and an EAM region (10 720 atoms). The lattice constant is compressed and expanded to simulate hydrostatic pressures and tensions, respectively. The convergence accuracy of the total energy is chosen as $5 \times 10^{-5} \mathrm{eV}$ in the relaxation of the electronic degrees of freedom. The relaxation for all of the atoms is performed until the maximum force on every atom is less than $0.01 \mathrm{eV} / \AA$.

We use constant pressure boundary conditions to calculate the dynamical matrix,

$$
D_{i, j}^{\alpha, \beta}=\frac{1}{m} \frac{\partial^{2} E}{\partial u_{i}^{\alpha} \partial u_{j}^{\beta}},
$$

under the harmonic approximation, where $m$ is the mass of atoms and $u_{i}^{\alpha}$ and $u_{j}^{\beta}$ are the displacements of atoms $i$ and $j$ in the directions $\alpha$ and $\beta$ from its relaxed equilibrium position, respectively. The formation entropy is evaluated via the following equation: ${ }^{22,24}$

$$
S_{n v}^{F}=-k_{B}\left(\sum_{i}^{3(N-n-1)} \ln \omega_{i}^{n v}-\frac{N-n-1}{N-1} \sum_{i}^{3(N-1)} \ln \omega_{i}^{0}\right),
$$

where $\omega_{i}^{n v}$ and $\omega_{i}^{0}$ represents the harmonic vibrational frequencies for the system with and without vacancies, respectively. Three acoustic phonon frequencies are removed from the sum owing to the translational invariance of the system. In the present work, we used the empirical force-matching EAM potential ${ }^{30}$ rather than the multiscale method to evaluate the entropy terms. This approach is justified because EAM is known to describe the elastic properties (the harmonic approximation of phonons corresponds to elastic deformations) of materials very well-EAM potentials are usually fitted to have excellent elastic constants. The supercell of the EAM calculation of vibrational entropy is chosen as $6 a_{0} \times 6 a_{0} \times 6 a_{0}$. The dynamical matrix is determined by displacing the atoms by $\pm 0.053 \AA$ from their relaxed equilibrium positions in all three Cartesian directions. ${ }^{24}$ The dynamical matrix is diagonalized and the eigenfrequencies are obtained to evaluate the formation entropy in Al.

We also calculate the formation energy of a monovacancy and a divacancy for other fcc metals, including $\mathrm{Cu}, \mathrm{Ag}, \mathrm{Au}$, $\mathrm{Ir}, \mathrm{Pt}, \mathrm{Ni}$, and $\mathrm{Pd}$, by using the Vienna $a b$ initio simulation package. ${ }^{34-36}$ The periodic supercell consists of 108 atoms $\left(3 a_{0} \times 3 a_{0} \times 3 a_{0}\right)$, which is sufficient to get converged vacancy formation energies. ${ }^{24,25}$ Large plane-wave cutoffs of 250 and $380 \mathrm{eV}$ are used for $(\mathrm{Ag}, \mathrm{Au}, \mathrm{Ir}, \mathrm{Pt})$ and $(\mathrm{Cu}, \mathrm{Ni}$, $\mathrm{Pd})$, respectively. The $k$ points are sampled according to the Monkhorst-Pack method ${ }^{37}$ with a $4 \times 4 \times 4 k$ mesh. The calculations are performed by using the highly accurate fullpotential projected augmentation wave method ${ }^{38,39}$ and the generalized gradient approximation in the parametrization by Perdew and Wang ${ }^{40}$ (PW91).

\section{RESULTS AND DISCUSSION}

Figure 1 shows the binding energy and Gibbs binding free energy for $n$-vacancy clusters ( $n$ ranges from 2 to 10 ) as a 

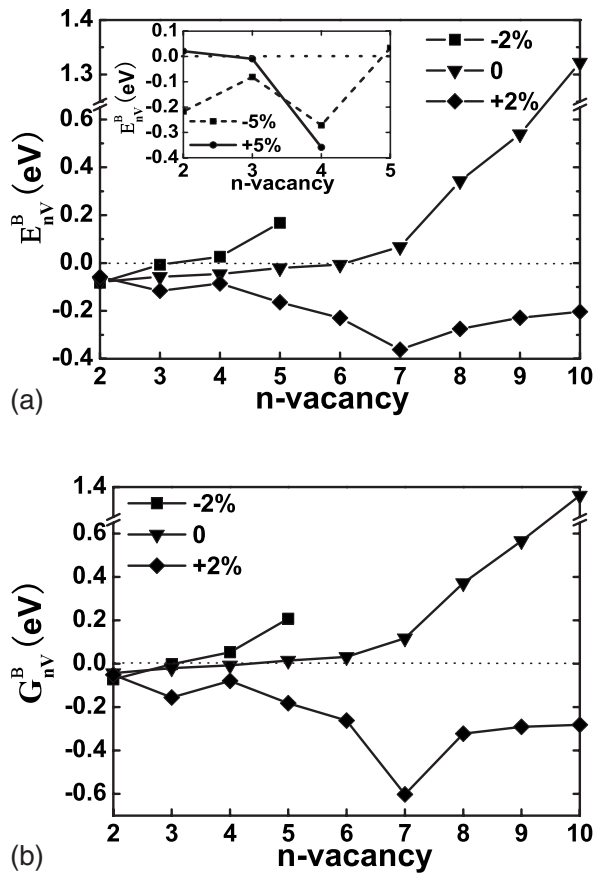

FIG. 1. Binding energy and Gibbs binding free energy of $n$-vacancy clusters in Al. $0, \pm 2 \%$, and $\pm 5 \%$ represent the percentage changes in lattice constant. (a) Binding energy versus number $n$. Inset: binding energy under a greater stress $( \pm 5 \%)$. (b) Gibbs binding free energy versus number $n$ at $300 \mathrm{~K}$.

function of hydrostatic pressures. At zero pressure, the binding energy of a divacancy in $\mathrm{Al}$ from the present multiscale calculation is $-0.08 \mathrm{eV}$, which is the same value as that obtained from a stand-alone Kohn-Sham DFT calculation. $^{23,24} \mathrm{~A}$ stand-alone OFDFT calculation gives a value of $-0.086 \mathrm{eV}^{41}$

\section{A. Dissociation probability of vacancy clusters}

In the following, we consider the thermal stability of vacancy clusters from the Gibbs free energy analysis. In thermal equilibrium, the concentration of an $n$-vacancy cluster is given by

$$
c_{n v}^{e}=\frac{g_{n v}}{n} e^{-G_{n v}^{F} / k_{B} T},
$$

where $g_{n v}$ is the coordination number of the vacancy cluster. ${ }^{42}$ Under the condition of a constant supersaturation, the steady-state concentration $c_{n v}^{s}$ is given by ${ }^{20} c_{n v}^{s}$ $=c_{n v}^{e}\left(S_{1}\right)^{n}$, where the concentration of supersaturated monovacancies is given as $S_{1}=c_{v}^{s} / c_{v}^{e}$. It turns out that the above two formulas yield the same results in terms of the relative stability of vacancy clusters; therefore, we omit the superscripts $e$ and $s$ in the following discussions. There are many different ways that a larger vacancy cluster can thermally dissociate into smaller ones. The total probability of dissociation, $P_{\text {tot }}(n)$, can be expressed as $P_{\text {tot }}=\Sigma_{i} P_{i}(n)$, where $P_{i}(n)$ represents the probability of a particular (the $i$ th) way of dissociation. For example, an $n$-vacancy cluster may simultaneously dissociate into $n$ monovacancies, the probability $P_{1}(n)$ of which is given by
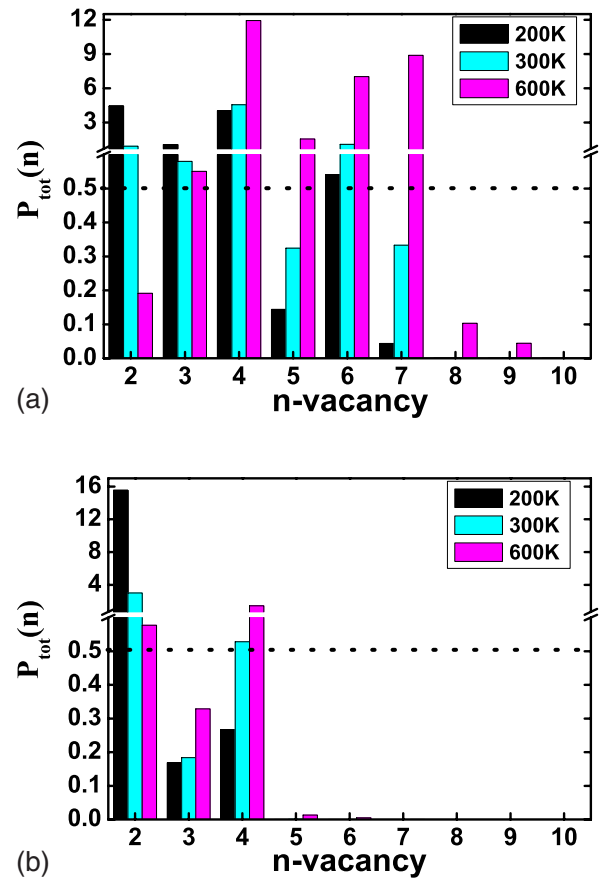

FIG. 2. (Color online) Calculated $P_{\text {tot }}(n)$ in the temperatures of 200,300 , and $600 \mathrm{~K}$ under (a) zero pressure and (b) compression of $-2 \%$.

$$
P_{1}(n)=\frac{\left(c_{1 v}\right)^{n}}{c_{n v}}=\frac{n}{g_{n v}} e^{-\left(n G_{1 v}^{F}-G_{n v}^{F}\right) / k_{B} T}=\frac{n}{g_{n v}} e^{-G_{n v}^{B} / k_{B} T},
$$

or an $n$-vacancy cluster could break into a monovacancy plus an $(n-1)$-vacancy cluster, the probability $P_{2}(n)$ of which is expressed as

$$
P_{2}(n)=\frac{c_{1 v} c_{(n-1) v}}{c_{n v}}=\frac{n g_{(n-1) v}}{(n-1) g_{n v}} e^{-\left(G_{n v}^{B}-G_{(n-1) v}^{B}\right) / k_{B} T} .
$$

To be more specific, let us consider the probability $P_{\text {tot }}(4)$ : We need to evaluate $P_{1}(4), P_{2}(4), P_{3}(4)$, and $P_{4}(4)$, and they represent the probabilities of dissociating a four-vacancy cluster into four monovacancies $(4=1+1+1+1)$ : a monovacancy and a three-vacancy cluster $(4=1+3)$, two divacancies $(4=2+2)$, and two monovacancies and a divacancy $(4=1$ $+1+2)$, respectively. Indeed, we have to consider all possible combinations in calculating a given $P_{\text {tot }}(n)$.

$P_{\text {tot }}(n)$, which is a key quantity in characterizing the tendency of void formation, is calculated for three different temperatures summarized in Fig. 2. We define the critical cluster size $n^{*}$ that satisfies the relation $P_{\text {tot }}(n)<0.5$ for all $n \geq n^{*}$. Therefore, $n^{*}$ represents the minimum nucleus size that is required for a cluster to grow into a void; once reaching the critical size, the vacancy cluster has a probability of more than $50 \%$ to survive and to grow into a void. While a negative $G_{n v}^{B}$ value renders a vacancy cluster unstable [i.e., $\left.P_{\text {tot }}(n)>0.5\right]$, a positive and rapidly increasing $G_{n v}^{B}$ as a function of $n$ is necessary for a stable void formation [cf. Eqs. (9) and (10)]. The calculation of $P_{\text {tot }}(n)$ reveals that $n^{*}=8$ at $T$ $=600 \mathrm{~K}$ and $n^{*}=7$ at $T=300$ and $200 \mathrm{~K}$ under zero pressure. The results suggest that, in equilibrium, voids are unlikely to 

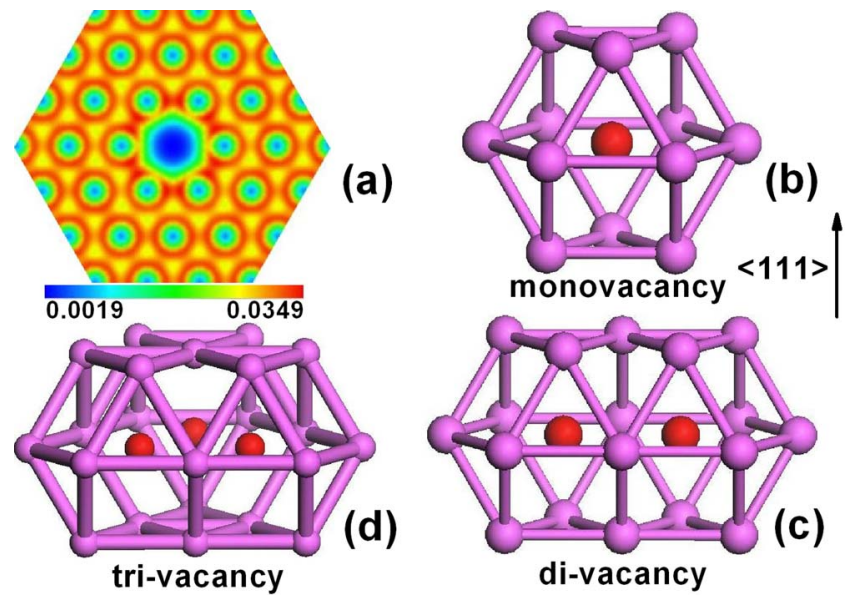

FIG. 3. (Color online) Charge density and schematic diagrams of bonding cages in Al. (a) Electron density $\left(\AA^{-3}\right)$ in a close-packed $\{111\}$ plane in the presence of a monovacancy. The vacancy induces accumulation of charge density (red ring) between the atoms in the first shell around the vacancy. The bonding ring is responsible for the formation of a bonding cage surrounding an $n$-vacancy cluster. The schematic diagrams of bonding cages surrounding (b) a monovacancy, (c) a divacancy, and (d) a trivacancy. The red spheres represent vacancies; the pink vertices and edges of the cage represent $\mathrm{Al}$ atoms and their nearest-neighbor bonds.

form in $\mathrm{Al}$. With a hydrostatic pressure of $-2 \%, n^{*}$ decreases to 5 at $600 \mathrm{~K}$. On the other hand, with a hydrostatic tension of $+2 \%, n^{*}>10$ because $G_{n v}^{B}$ is negative for all $n<10$. We also performed the calculations for a greater compression and/or tension of $\pm 5 \%$, and we find the same trend as in the case of $\pm 2 \%$. Therefore, applying a hydrostatic pressure can facilitate void formation, and a tension can inhibit or even prohibit void formation. In conclusion, a positive and rapidly increasing $G_{n v}^{B}$ function is a necessary and sufficient condition for a stable void formation. It is clear from Fig. 1 that the binding energy dominates the Gibbs binding free energy; therefore, the binding energy is the central physical quantity that determines the thermodynamic stability of vacancy clusters. In the following, we will explore the electronic origin of void formation in fcc metals by examining how the electronic structure influences the binding energy.

\section{B. Bonding cages of vacancy clusters}

In Fig. 3(a), we present the electron charge density plot in a $\{111\}$ plane of $\mathrm{Al}$ in the presence of a monovacancy. We find that there is charge accumulation (or a bonding ring) formed between the atoms in the first shell around the vacancy. This vacancy-induced bonding (or the strengthening of bonding) was also observed by others. ${ }^{23,25}$ Because there are three equivalent $\{111\}$ planes in an fcc crystal, the four equivalent bonding rings constitute a bonding cage surrounding the monovacancy, as shown in Fig. 3(b). We refer to the vacancy-induced bonding as "cage bonds." The same bonding cages are also observed for an $n$-vacancy cluster with a small $n$ (e.g., $n=2,3,4, \ldots$ ) from the electronic structure calculations. ${ }^{43}$ The schematic pictures for a divacancy and a trivacancy are shown in Figs. 3(c) and 3(d), respectively. In addition, we find that for a smaller $n$, the atomic relaxation is negligible, while for a larger $n$, the relaxation is significant. For example, the maximum atomic displacement is $d_{\max }$ $=0.075 \AA$ for a divacancy and $d_{\max }=1.657 \AA$ for a tenvacancy cluster.

Next, we present a simple model that captures the essential physics of vacancy cluster formation. The formation energy of an $n$-vacancy cluster can be roughly divided into two contributions. One is the energy required to break the nearest-neighbor interatomic bonds, which can be written as $N_{n} \Delta_{b}$; here, $N_{n}$ refers to the number of broken interatomic bonds and $\Delta_{b}$ is the energy per bond. The other contribution is the energy released by both electronic relaxation (e.g., the formation of a bonding cage), $\Delta_{f}(n)$, and atomic relaxation, $\Delta_{r}(n)$, upon forming a vacancy cluster. Thus, we can express the formation energy of a vacancy cluster as $E_{n v}^{F}=N_{n} \Delta_{b}$ $-\left[\Delta_{f}(n)+\Delta_{r}(n)\right]$. Accordingly, the binding energy can be derived as

$$
E_{n v}^{B}=\left(n N_{1}-N_{n}\right) \Delta_{b}-\left[n \Delta_{f}(1)-\Delta_{f}(n)\right]+\left[\Delta_{r}(n)-n \Delta_{r}(1)\right],
$$

where $N_{1}, \Delta_{f}(1)$, and $\Delta_{r}(1)$ represents the corresponding physical quantity for a monovacancy. For a larger $n, \Delta_{r}(n)$ dominates all of the other energy contributions in Eq. (11), and thus, the binding energy monotonously increases with respect to $n$ (see Fig. 1). This is the reason why we only need to consider $n$ up to 10 in the binding energy calculations. For a smaller $n$, one can ignore the atomic relaxation energy, and thus, the binding energy becomes

$$
E_{n v}^{B} \simeq\left(n N_{1}-N_{n}\right) \Delta_{b}-\left[n \Delta_{f}(1)-\Delta_{f}(n)\right] .
$$

It is important to note that the formation of the bonding cage is an indication of a strong electronic relaxation, hence a considerable contribution of the second term in Eq. (12). By a simple bond counting, one finds that there is a net loss of ten cage bonds but a net gain of one interatomic bond upon merging two monovacancies into a divacancy. Since the energy loss due to the cage bonds exceeds the energy gain of the interatomic bond, the binding energy is negative according to Eq. (12), and therefore, the divacancy is energetically unstable in $\mathrm{Al}$.

\section{General trend of void formation in fcc metals}

The analysis of Sec. III B strongly suggests that the bonding cage plays a crucial role in the formation of vacancy clusters. Motivated by the observation in $\mathrm{Al}$, we performed additional $a b$ initio calculations for other fcc metals including $\mathrm{Cu}, \mathrm{Ag}$, Au, Ir, Pt, Ni, and Pd. As shown in Table I, it is found that the binding energy of a divacancy is positive for $\mathrm{Cu}, \mathrm{Ni}, \mathrm{Pd}$, and $\mathrm{Ag}$ (group I) and negative for Ir, Pt, and $\mathrm{Au}$ (group II, including Al). Figure 4 shows the bonding charge density $\rho_{\text {bond }}(\mathbf{r})$ for a monovacancy, which is defined as

$$
\rho_{\text {bond }}(\mathbf{r})=\rho^{\text {solid }}(\mathbf{r})-\sum_{i} \rho^{\text {atom }}\left(\mathbf{r}-\mathbf{R}_{i}\right) .
$$

Here, $\rho^{\text {solid }}$ is the charge density calculated for a periodic solid in a unit cell, and $\rho^{\text {atom }}\left(\mathbf{r}-\mathbf{R}_{i}\right)$ represents the atomic 
TABLE I. Calculated values of monovacancy formation energy $E_{v}^{F}$ (in eV), divacancy binding energy $E_{2 v}^{B}$ (in eV), and percentage change of force constant upon forming a monovacancy. Here, $\varphi_{B}$ and $\varphi_{v}$ represent the force constant between the nearest-neighbor atoms in the perfect lattice and that in the first shell around the monovacancy, respectively.

\begin{tabular}{lcccccccc}
\hline \hline & $\mathrm{Ir}$ & $\mathrm{Pt}$ & $\mathrm{Au}$ & $\mathrm{Al}$ & $\mathrm{Ag}$ & $\mathrm{Pd}$ & $\mathrm{Ni}$ & $\mathrm{Cu}$ \\
\hline$E_{v}^{F}$ & $1.87\left(1.88^{\mathrm{a}}\right)$ & $1.02\left(1.35^{\mathrm{b}}\right)$ & $0.75\left(0.93^{\mathrm{b}}\right)$ & $0.61\left(0.67^{\mathrm{b}}\right)$ & $1.10\left(1.11^{\mathrm{b}}\right)$ & $1.51\left(1.70^{\mathrm{b}}\right)$ & $1.66\left(1.79^{\mathrm{b}}\right)$ & $1.25\left(1.28^{\mathrm{b}}\right)$ \\
$E_{2 v}^{B}$ & -0.32 & -0.30 & -0.15 & $-0.08\left(-0.08^{\mathrm{c}}\right)$ & 0.003 & 0.01 & 0.04 & $0.07\left(0.08^{\mathrm{d}}\right)$ \\
$\frac{\varphi_{v}-\varphi_{B}}{\varphi_{B}}(\%)$ & 28 & 27 & 24 & $22\left(25^{\mathrm{c}}\right)$ & 13 & 12 & 9 & 10 \\
\hline \hline
\end{tabular}

${ }^{\mathrm{a}}$ Computational data from Ref. 44.

${ }^{b}$ Experimental data from Ref. 45.

${ }^{\mathrm{c} C o m p u t a t i o n a l ~ d a t a ~ f r o m ~ R e f . ~} 24$.

${ }^{\mathrm{d}}$ Computational data from Ref. 21.

charge density centered at the $i$ th ion in the same unit cell. The sum is over all ions in the unit cell. We have also evaluated the nearest-neighbor force constant matrix $\varphi_{i, j}^{\alpha, \beta}$ $=m D_{i, j}^{\alpha, \beta}$ in the absence and presence of a monovacancy in all of the fcc metals studied here. For the latter case, $\varphi_{i, j}^{\alpha, \beta}$ is calculated between the nearest-neighbor atoms $i$ and $j$ in the first shell around the vacancy. The force constant matrix $\varphi_{i, j}^{\alpha, \beta}$ is a $3 \times 3$ matrix for the fixed $i$ and $j$, and the largest eigenvalue of the matrix $\varphi_{i, j}^{\alpha, \beta}$ corresponds to the force constant along the line joining the two atoms $i$ and $j$.

Since the formation of a bonding cage is an indication of a significant electronic relaxation around a vacancy, the force constant should increase upon the formation of the bonding cage-this, indeed, is the case, as clearly shown in Table I. The percentage increase in the force constant in group II metals doubles that in group I metals. In conclusion, we find a one-to-one correspondence between the negative binding energy and the formation of a bonding cage. The latter is the result of a significant electronic relaxation upon vacancy formation. This is an important result because it shows that the essential physics for void formation is the same for all fcc metals.

We have primarily attributed the thermodynamic stability $\left(P_{\text {tot }}\right.$ and $\left.n^{*}\right)$ of vacancy clusters to the vacancy binding en- ergy, which could be understood from a bonding cage model via Eqs. (11) and (12). For group I, because the second term in Eq. (12) is negligible compared to the first term and $\left(n N_{1}-N_{n}\right)$ is an increasing function of $n$, the binding energy $E_{n v}^{B}$ is positive and increasing. Therefore, voids can be formed more easily in group I metals based on the analysis of $P_{\text {tot }}$ and $n^{*}$. For group II, the second term may overtake the first term for a smaller $n$ and offset its increase for a larger $n$. Therefore, the formation of the bonding cage results in a negative or a slowly increasing binding energy, which inhibits void formation. Since the critical cluster size $n^{*}$ is in the range where Eq. (12) holds, one concludes that there is a prominent connection between the formation of a bonding cage and the tendency of void formation in fcc metals: the formation of a bonding cage hinders void formation and the absence of a bonding cage implies a stronger tendency of void formation. It was experimentally observed that the incubation time of void formation is increased and the void concentration is decreased when $\mathrm{Al}$ concentration in a disordered NiAl alloy is increased. ${ }^{1}$ This observation is consistent with the general trend that we have predicted in this work. Finally, from Fig. 4, it is evident that the bonding cage is formed in fcc metals whose atomic bonding is of more directional character. This may be explained by the observation that the drastic rearrangement of electron distribution upon
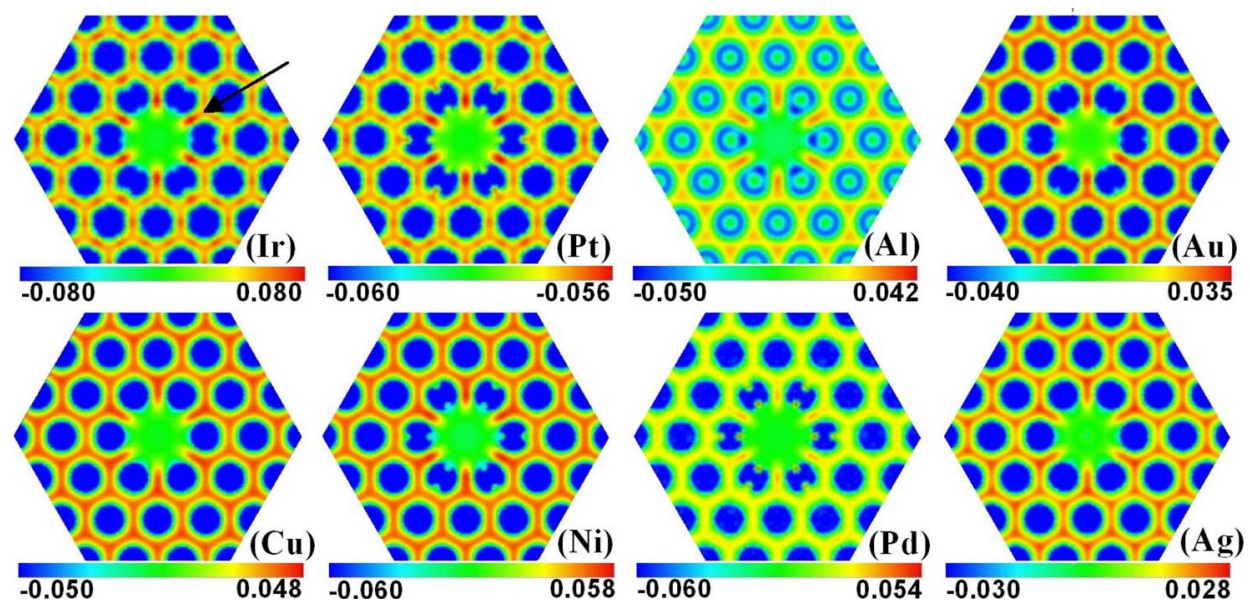

FIG. 4. (Color online) Bonding charge density $\left(\AA^{-3}\right)$ in a close-packed $\{111\}$ plane for a series of fcc metals in the presence of a monovacancy. An additional bonding (stronger red color) between the atoms in the first shell of the vacancy is clearly visible in Ir, Pt, Al, and $\mathrm{Au}$, which have stronger directional bondings. The bonding ring is very weak or nonexistent in $\mathrm{Cu}, \mathrm{Ni}, \mathrm{Pd}$, and $\mathrm{Ag}$, wherein the bondings are less directional or more metalliclike. The bonding ring of Ir is indicated by an arrow. The bonding ring can be found at the same location in other group II materials. 
perturbation, such as the formation of a bonding cage, is often associated with directional bonding.

\section{CONCLUSION}

In summary, we have studied the electronic origin of void formation in fcc metals from thermodynamic analysis and electronic structure calculations. The critical nucleus size for void formation in $\mathrm{Al}$ is determined as a function of temperature and pressure. While a hydrostatic pressure can enhance void formation, a tension could lead to the opposite effect. We discover that the bonding cage plays a crucial role in void formation of all fcc metals. A model proposed to elucidate the connection between the bonding cage and the Gibbs free energy suggests that the formation of the bonding cage leads to a negative and slowly increasing Gibbs free energy; this, in turn, reduces the propensity of void formation. On the other hand, fcc metals that do not exhibit bonding cages are more susceptible to void formation. Finally, we find that the directionality of atomic bonding in fcc metals is responsible for the formation of the bonding cage.

\section{ACKNOWLEDGMENTS}

The authors wish to thank Efthimios Kaxiras, Sadasivan Shankar, and Nasr Ghoniem for fruitful discussions. This work was supported in part by Intel Corporation, NSF PREM Grant No. DMR-0611562, and DOE SciDAC Grant No. DE-FC02-06ER2579.
*Corresponding author; ganglu@ csun.edu

${ }^{1}$ Effects of radiaion on substructure and mechanical properties of metals and alloys, edited by J. Moteff, ASTM STP No. 529 (American Society for Testing and Materials, Philadelphia, 1973).

${ }^{2}$ M. H. Hassan, J. P. Blanchard, and G. L. Kulcinski, StressEnhanced Swelling: Mechanisms and Implication for Fusion Reactors (University of Wisconsin, Madison, WI, 1992).

${ }^{3}$ R. J. Gleixner and W. D. Nix, J. Appl. Phys. 86, 1932 (1999).

${ }^{4}$ J. Cho, M. R. Gungor, and D. Maroudas, Appl. Phys. Lett. 86, 241905 (2005).

${ }^{5}$ E. T. Seppälä, J. Belak, and R. E. Rudd, Phys. Rev. Lett. 93, 245503 (2004).

${ }^{6}$ K. F. Kelton, in Solid State Physics, edited by H. Ehrenreich and D. Turnbull (Academic, New York, 1991), Vol. 45, pp. 75-177.

${ }^{7}$ D. Kashchiev, Nucleation: Basic Theory with Applications (Butterworth Heinemann, Oxford, 2000).

${ }^{8}$ F. Soisson and G. Martin, Phys. Rev. B 62, 203 (2000).

${ }^{9}$ F. F. Abraham, Homogeneous Nucleation Theory (Academic, New York, 1974).

${ }^{10}$ D. W. Oxtoby, J. Phys.: Condens. Matter 4, 7627 (1992).

${ }^{11}$ E. Clouet and M. Nastar, Phys. Rev. B 75, 132102 (2007).

${ }^{12}$ H. Mehl, O. Biham, O. Millo, and M. Karimi, Phys. Rev. B 61, 4975 (2000).

${ }^{13}$ D. J. Bacon, A. F. Calder, and F. Gao, J. Nucl. Mater. 251, 1 (1997)

${ }^{14}$ J. M. Soler, Phys. Rev. B 53, R10540 (1996).

${ }^{15}$ N. Tajima, O. Takai, Y. Kogure, and M. Doyama, Comput. Mater. Sci. 14, 152 (1999).

${ }^{16}$ N. Choly, G. Lu, E. Weinan, and E. Kaxiras, Phys. Rev. B 71, 094101 (2005).

${ }^{17}$ X. Zhang and G. Lu, Phys. Rev. B 76, 245111 (2007).

${ }^{18}$ J. L. Katz and H. Wiedersich, J. Chem. Phys. 55, 1414 (1971).

${ }^{19}$ C. F. Clement and M. H. Woods, J. Nucl. Mater. 89, 1 (1980).

${ }^{20}$ R. A. Johnson, Phys. Rev. B 28, 6695 (1983).

${ }^{21}$ P. A. Korzhavyi, I. A. Abrikosov, B. Johansson, A. V. Ruban, and H. L. Skriver, Phys. Rev. B 59, 11693 (1999).

${ }^{22}$ Y. Mishin, M. R. Sorensen, and A. F. Voter, Philos. Mag. A 81, 2591 (2001).

${ }^{23}$ K. Carling, G. Wahnström, T. R. Mattsson, A. E. Mattsson, N.
Sandberg, and G. Grimvall, Phys. Rev. Lett. 85, 3862 (2000).

${ }^{24}$ K. M. Carling, G. Wahnström, T. R. Mattsson, N. Sandberg, and G. Grimvall, Phys. Rev. B 67, 054101 (2003).

${ }^{25}$ T. Uesugi, M. Kohyama, and K. Higashi, Phys. Rev. B 68, 184103 (2003).

${ }^{26}$ R. D. Hatcher, R. Zeller, and P. H. Dederichs, Phys. Rev. B 19, 5083 (1979).

${ }^{27}$ P. Garcia-Gonzalez, J. E. Alvarellos, and E. Chacon, Phys. Rev. B 53, 9509 (1996).

${ }^{28}$ L. W. Wang and M. P. Teter, Phys. Rev. B 45, 13196 (1992).

${ }^{29}$ Y. A. Wang, N. Govind, and E. A. Carter, Phys. Rev. B 60, 16350 (1999).

${ }^{30}$ F. Ercolessi and J. Adams, Europhys. Lett. 26, 583 (1994).

${ }^{31}$ Y. Liu, G. Lu, Z. Chen, and N. Kioussis, Modell. Simul. Mater. Sci. Eng. 15, 275 (2007).

${ }^{32}$ L. Goodwin, R. J. Needs, and V. Heine, J. Phys.: Condens. Matter 2, 351 (1990).

${ }^{33}$ J. P. Perdew and A. Zunger, Phys. Rev. B 23, 5048 (1981).

${ }^{34}$ G. Kresse and J. Hafner, Phys. Rev. B 47, 558 (1993).

${ }^{35}$ G. Kresse and J. Furthmuller, Phys. Rev. B 54, 11169 (1996).

${ }^{36}$ G. Kresse and J. Furthmuller, Comput. Mater. Sci. 6, 15 (1996).

${ }^{37}$ H. J. Monkhorst and J. D. Pack, Phys. Rev. B 13, 5188 (1976).

${ }^{38}$ P. E. Blöchl, Phys. Rev. B 50, 17953 (1994).

${ }^{39}$ G. Kresse and D. Joubert, Phys. Rev. B 59, 1758 (1999).

${ }^{40}$ J. P. Perdew, in Electronic Structure of Solids, edited by P. Ziesche and H. Eschrig (Akademie, Berlin, 1991).

${ }^{41}$ G. Ho, M. T. Ong, K. J. Caspersen, and E. A. Carter, Phys. Chem. Chem. Phys. 9, 4951 (2007).

${ }^{42}$ G. Grimvall, Thermophysical Properties of Materials (Elsevier, Amsterdam, 1999).

${ }^{43}$ The bonding "cage" is also observed in a larger vacancy cluster although the cage is significantly distorted owing to the much greater atomic relaxation.

${ }^{44}$ Y. N. Gornostyrev, M. I. Katsnelson, N. I. Medvedeva, O. N. Mryasov, A. J. Freeman, and A. V. Trefilov, Phys. Rev. B 62, 7802 (2000).

${ }^{45}$ H. Schultz and P. Ehrhart, in Atomic Defects in Metals, LandoltBörnstein, New Series, Group III, edited by H. Ullmaier (Springer, Berlin, 1991). 